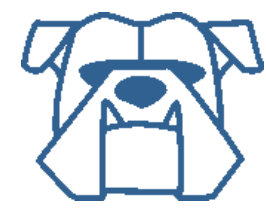

Kettering University Digital Commons @ Kettering University

\title{
Nanoparticle aggregation and relaxation effects in ferrofluids: studied through anisotropic light scattering
}

Corneliu Rablau

Kettering University

Prem Vaishnava

Kettering University

Sudakar Chandran

Wayne State University

Ronald Tackett

Wayne State University

Gavin Lawes

Wayne State University

See next page for additional authors

Follow this and additional works at: https://digitalcommons.kettering.edu/physics_facultypubs Part of the Physics Commons

\section{Recommended Citation}

Rablau, Corneliu; Vaishnava, Prem; Chandran, Sudakar; Tackett, Ronald; Lawes, Gavin; and Naik, Ratna, "Nanoparticle aggregation and relaxation effects in ferrofluids: studied through anisotropic light scattering" (2008). Physics Publications. 38.

https://digitalcommons.kettering.edu/physics_facultypubs/38 
Authors

Corneliu Rablau, Prem Vaishnava, Sudakar Chandran, Ronald Tackett, Gavin Lawes, and Ratna Naik 


\title{
Nanoparticle aggregation and relaxation effects in ferrofluids studied through anisotropic light scattering
}

\author{
Corneliu Rablau*a, Prem Vaishnava ${ }^{\mathrm{a}}$, Chandran Sudakar ${ }^{\mathrm{b}}$, Ronald Tackett ${ }^{\mathrm{b}}$, Gavin Lawes ${ }^{\mathrm{b}}$, Ratna \\ Naik $^{\mathrm{b}}$ \\ ${ }^{a}$ Department of Physics, Kettering University, 1700 West Third Ave., Flint, MI, USA 48504; \\ ${ }^{\mathrm{b}}$ Department of Physics and Astronomy, Wayne State University, Detroit, MI, USA 48201
}

\begin{abstract}
We have investigated the aggregation and dissociation dynamics of 6-nm size $\mathrm{Fe}_{3} \mathrm{O}_{4}$ nanoparticles coated by tetra methyl ammonium hydroxide (TMAH) and the same size $\gamma-\mathrm{Fe}_{2} \mathrm{O}_{3}$ nanoparticles precipitated inside an alginate hydrogel matrix, both in aqueous suspensions, using dc magnetic-field-induced time-dependent light scattering patterns. For the $\mathrm{Fe}_{3} \mathrm{O}_{4}$ ferrofluid, a strong anisotropy in light scattering was observed for light propagating perpendicular to the magnetic field. This behavior is attributed to the aggregation of the nanoparticles into chain-like and column-like structures oriented parallel to the magnetic field. A significantly different behavior is observed for the aqueous suspension of $\gamma-\mathrm{Fe}_{2} \mathrm{O}_{3}$ nanoparticles precipitated in alginate hydrogel, for which the application of the dc magnetic field produced little to no change in the light scattering patterns. We attribute this difference to the constrained random distribution of $\gamma-\mathrm{Fe}_{2} \mathrm{O}_{3}$ nanoparticles precipitated in the alginate matrix. Correlating the results from this investigation with our previous study of magneto-thermal measurements in ac fields [Vaishnava et al., J. Appl. Phys. 102, 063914 (2007)], we conclude that for a ferrofluid to exhibit significant thermal effects under an ac magnetic field, it should exhibit optical anisotropy by developing a chain like structure under the influence of a dc magnetic field.
\end{abstract}

Keywords: Ferrofluid, nanoparticles, field-induced optical anisotropy, chain formation, time-dependent, $\mathrm{Fe}_{3} \mathrm{O}_{4}, \gamma-\mathrm{Fe}_{2} \mathrm{O}_{3}$

\section{INTRODUCTION}

Ferrofluids consist of colloidal suspensions of coated superparamagnetic nanoparticles dispersed in a carrier liquid. These field-controllable fluids have been studied in recent years for their potential technological and biomedical applications. Notable biomedical applications include the use of ferrofluids as a targeted delivery vehicle for therapeutic agents $^{1}$, as contrast enhancement agents in Magnetic Resonance Imaging, as well as for hyperthermia locoregional treatment in tumor therapy ${ }^{2}$. A thorough knowledge and understanding of the behavior of ferrofluids in dc and ac magnetic fields is essential for their successful incorporation in these applications. In particular, the formation of clusters and structures in a ferrofluid under a dc magnetic field has attracted much attention in the past decade, due to the impact these structures have on the physical properties of the fluid. It has been shown that when a magnetic field is applied to a ferrofluid, the particles in suspension will typically form chainlike and column-like structures oriented preferentially parallel to the magnetic field..$^{3-12}$ The structures are the result of the interaction between the field-oriented magnetic moments of the nanoparticles and their subsequent aggregation through diffusion. This orientation occurs through two possible mechanisms: either the particle rotates altogether with its magnetic moment (Brownian rotation), or the particle maintains its physical orientation, and the magnetic moment vector rotates within the nanoparticle, to align with the field (Neel relaxation). ${ }^{13}$ The relative contributions of these two mechanisms will have a significant impact on determining the structures formed under a dc magnetic field. These mechanisms also strongly affect the properties of the ferrofluid under an ac magnetic field, including magnetic dissipative loss, which is relevant for hyperthermia applications.

The formation of dc field-induced structures in field-controllable fluids and their effect on the properties of the suspension have been studied theoretically $y^{14-19}$ and experimentally through Raman scattering, ${ }^{20}$ birefringence and scattering dichroism measurements, ${ }^{10,11,21}$ Faraday rotation and Faraday ellipticity, ${ }^{22,}{ }^{23}$ visible light and/or x-ray scattering, ${ }^{7,11,12,24,25}$ and optical attenuation or transmission measurements. ${ }^{4,9,26}$ The current model for pattern

*crablau@kettering.edu; phone $1810762-7867$

Plasmonics: Metallic Nanostructures and Their Optical Properties VI, edited by Mark I. Stockman Proc. of SPIE Vol. 7032, 70320Z, (2008) · 0277-786X/08/\$18 · doi: 10.1117/12.791987 
formation in ferrofluids consists of a two-stage process: chain formation and column formation. In this framework, the nanoparticles, acting as magnetic dipoles, first orient and aggregate along field lines to form chains. At longer times, the chains aggregate through lateral interaction to form columns, which further aggregate into even thicker columns. This theory, first developed by Halsey and Toor $(\mathrm{HT})^{3}$ for electrorheological fluids, was further refined and extended to magnetic fluids by Martin et al., ${ }^{4}$ who identified thermal fluctuations and topological defects as the two main mechanisms for lateral interactions and chain coalescence. This refined theory was tested by Furst and Gast ${ }^{6}$ through video microscopy and direct measurement of the lateral interaction between dipolar chains using optical trapping, who found that lateral interactions leading to chain coalescence into columns govern the long time evolution of the structures in suspension. The dominant mechanism for chain coalescence was found to depend on concentration, the strength of the dipolar interaction, and the particle polydispersity and roughness.

In spite of the relatively large number of studies cited above, data dealing with the kinematics of field-induced aggregation, chain-formation and coarsening in ferrofluids, as well as the kinematics of the corresponding dissociation processes upon removal of the magnetic field are scarce. Indeed, most of the studies performed to date on the time evolution of the chain and cluster formation and dissociation have involved electrorheological or magnetorheological fluids, where the size of the dispersed phase was in the micrometer range. ${ }^{4,5,10,11}$ Furthermore, the few time-dependent optical transmission or scattering measurements that have been reported have used a single light beam, which limits the information about the chain kinematics to only one orthogonal projection of a three-dimensional process. Likewise, there are few data available on the correlation between structural response of a ferrofluid and the relative contributions of the Brownian and Neel relaxation. Recently, we have investigated the time-dependent field-induced light scattering patterns produced by two orthogonal light beams through an aqueous suspension of $\mathrm{Fe}_{3} \mathrm{O}_{4}$ nanoparticles coated with tetramethyl ammonium hydroxide (TMAH) under the OFF-ON transient of a dc magnetic field. ${ }^{12}$ We discussed the results of these experiments in the framework set by Kruse et al. ${ }^{7}$ in their 2D small angle x-ray scattering (SAXS) study of field-induced anisotropy in ferrofluids. From the time evolution of the anisotropic scattering patterns produced by the $\mathrm{Fe}_{3} \mathrm{O}_{4}$ ferrofluid we extracted the time dependence of the field-induced optical anisotropy, which we proposed can be explained within the framework of the two-stage chain formation and coarsening process introduced by Halsey and Toor ${ }^{3}$ and expanded by Martin et al., ${ }^{4}$ and Furst and Gast ${ }^{6}$.

In this paper we extend the scope of this previous investigation to a comparative study of two different ferrofluid systems, and specifically investigate the dynamics in these two systems under both the $O F F-O N$ and $O N-O F F$ transients of a dc magnetic field. The first system is the aqueous suspension of relatively free and isolated TMAHsurfacted $\mathrm{Fe}_{3} \mathrm{O}_{4}$ (magnetite) nanoparticles employed in our previous study; the second is an aqueous suspension of $\gamma$ $\mathrm{Fe}_{2} \mathrm{O}_{3}$ (maghemite) nanoparticles precipitated in alginate hydrogel matrix, in which the motion of the iron oxide nanoparticles (Brownian rotation as well as diffusion) is strongly constrained by the alginate matrix. We contrast the strong field-induced optical anisotropy observed in the $\mathrm{Fe}_{3} \mathrm{O}_{4}$ ferrofluid for both the $O F F-O N$ and $O N-O F F$ dc field transients with the lack of such field-induced optical anisotropy in the $\gamma-\mathrm{Fe}_{2} \mathrm{O}_{3}$ ferrofluid. Based on our previous work, ${ }^{12}$ we explain the time evolution of the anisotropic scattering patterns produced by the $\mathrm{Fe}_{3} \mathrm{O}_{4}$ ferrofluid through chain formation and coarsening during the $O F F-O N$ transition and the dissociation of those field-induced structures during the $O N-O F F$ transition. We attribute the differing optical response of the two systems to chain formation in the $\mathrm{Fe}_{3} \mathrm{O}_{4}$ ferrofluid and lack of such field induced structures in the $\gamma-\mathrm{Fe}_{2} \mathrm{O}_{3}$ ferrofluid because of the constrained random distribution of the nanoparticles in the alginate matrix. This interpretation is consistent with the results of our previous magneto-thermal (hyperthermia) measurements in ac magnetic fields ${ }^{27}$ where the magnetite ferrofluid exhibited strong Brownian relaxation near the freezing temperature, but no Brownian relaxation was observed for the $\gamma-\mathrm{Fe}_{2} \mathrm{O}_{3}$ ferrofluid.

\section{EXPERIMENTAL}

\subsection{Sample preparation}

The $\mathrm{Fe}_{3} \mathrm{O}_{4}$ ferrofluid sample was prepared by coprecipitation by mixing $4 \mathrm{~mL}$ of $1 \mathrm{M} \mathrm{FeCl}_{3}$ with $1 \mathrm{~mL}$ of $2 \mathrm{M} \mathrm{FeCl}_{2}$ solution then adding $50 \mathrm{~mL}$ of $1 \mathrm{M}$ aqueous ammonium hydroxide solution over a period of 5 minutes. $\mathrm{Fe}_{3} \mathrm{O}_{4}$ (magnetite) in the form of a black precipitate was formed which we allowed to settle at the bottom of a beaker. After decanting the clear liquid we washed the precipitate three times with de-ionized water and added $2 \mathrm{~mL}$ of $25 \%$ tetra methyl ammonium hydroxide to prevent agglomeration of the magnetite nanoparticles. Nanometer-size particles of $\gamma-\mathrm{Fe}_{2} \mathrm{O}_{3}$ were synthesized by cross-linking sodium alginate with $\mathrm{FeCl}_{3}$ and $\mathrm{FeCl}_{2}$ salts in methanol-water solution, as described in Ref. [28]. The resulting iron ions cross-linked alginate forms hydrogel beads, which are subsequently oxidized to form $\gamma$ $\mathrm{Fe}_{2} \mathrm{O}_{3}$ particles. After washing to remove excess iron, the entire synthesis process is repeated so that the further loading 
with the chloride salts increases the amount of iron oxide and the particle size. Prior to any physical characterization, the iron oxide hydrogels were dehydrated using a vacuum freeze dryer and crushed to obtain powder. For optical scattering measurements, the samples were suspended in de-ionized water to a volume fraction $\Phi$ of $1 \%$.

\subsection{Sample characterization}

The samples were characterized by X-ray Diffraction (XRD), Transmission Electron Microscopy (TEM) and SQUID magnetometer. The complete details of the characterization can be found in Ref. [27] and [29]. In brief, the XRD measurements showed the samples were a single phase crystalline with the expected structures for magnetite and maghemite. The distribution of particle sizes determined from the TEM measurements was fit with a Gaussian distribution and the average diameter of the $\mathrm{Fe}_{3} \mathrm{O}_{4}$ nanoparticles in the ferrofluid sample was determined to be $6 \pm 2 \mathrm{~nm}$. The particles size of $\gamma-\mathrm{Fe}_{2} \mathrm{O}_{3}$ particles was found to be $6 \pm 2 \mathrm{~nm}$ by XRD measurements. SQUID magnetic characterization showed superparamagnetic behavior for both samples at room temperature. The ac magneto-thermal (hyperthermia) measurements for the magnetite ferrofluid exhibited strong Brownian relaxation near the freezing temperature. No Brownian relaxation was observed for the $\gamma-\mathrm{Fe}_{2} \mathrm{O}_{3}$ ferrofluid and the rate of change of temperature for the meghamite ferrofluid was significantly lower than that of the magnetite ferrofluid ${ }^{27}$. These data are consistent with the suggestion that the $\mathrm{Fe}_{3} \mathrm{O}_{4}$ nanoparticles can diffuse freely through the carrier liquid while motion of the $\gamma$ - $\mathrm{Fe}_{2} \mathrm{O}_{3}$ nanoparticles is constrained by the alginate matrix.

\subsection{Light scattering measurements}

A schematic of the experimental setup used for the light scattering measurements is presented in Fig.1. The ferrofluid sample in a standard quartz suprasil spectrophotometer cuvette is placed at the center of a pair of water-cooled Helmholtz coils set. DC magnetic field values up to 400 Oe were used in these experiments, and the 3D mapping of the field revealed a field uniformity of better than $0.5 \%$ over the $\sim 2 \mathrm{~mL}$ volume of the ferrofluid sample. The light beams from two He-Ne lasers $(\lambda=632.8 \mathrm{~nm})$ were transmitted through the sample simultaneously, parallel and perpendicular to the applied magnetic field, respectively. The beam diameter through the sample was $\sim 1 \mathrm{~mm}$.

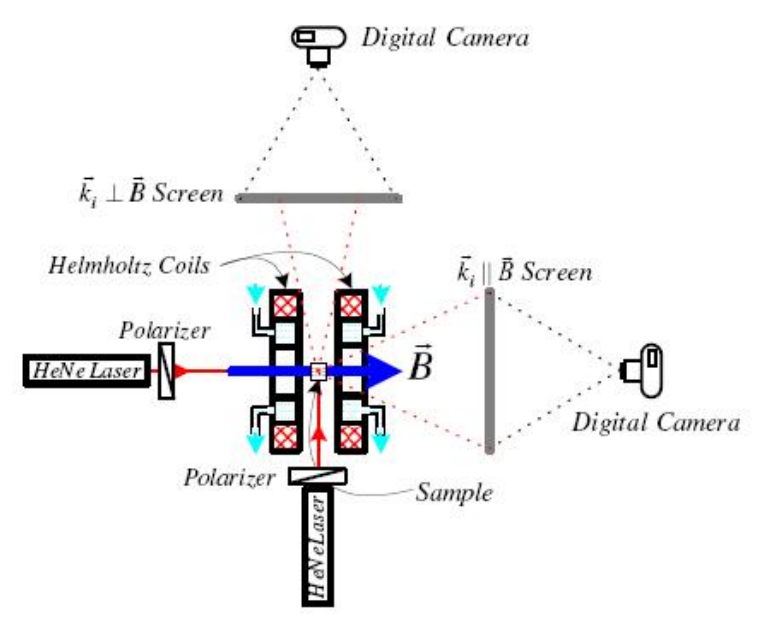

a)

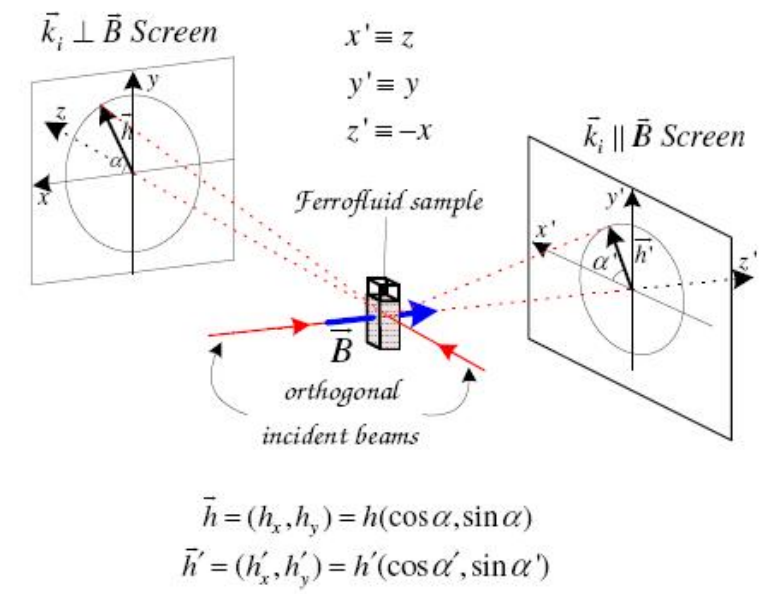

b)

Fig. 1.a) Schematic of the experimental setup for synchronous recording of the time-dependent light scattering patterns produced by two orthogonal light beams through a ferrofluid sample placed in a uniform magnetic field. b) The 3D geometry of the experiment. The scattered intensity on each screen is a function of the corresponding scattering vector $\vec{h}$ or $\vec{h}^{\prime}$.

Measurements were performed both with unpolarized and linearly polarized light having various polarization orientations with respect to the applied magnetic field. The incident laser power ranged from 1 to $10 \mathrm{~mW}$, and was the same for both lasers in any given experiment. The light path through the sample was $10 \mathrm{~mm}$ in both directions. The 
light scattered by the sample was projected onto two $220 \mathrm{~mm}$ x $220 \mathrm{~mm}$ translucent screens placed a distance of $500 \mathrm{~mm}$ from the center of the sample. To reflect their orientation relative to the magnetic field and to the propagation wave vector $\vec{k}_{i}$ of the incident light, we will call these the $\vec{k}_{i} \perp \vec{B}$ and $\vec{k}_{i} \| \vec{B}$ screens, respectively. This geometry combined with the dimensions and structure of the Helmholtz coils allowed scattering toward the $\vec{k}_{i} \| \vec{B}$ screen in a circular cone of half-angle $\sim 12^{\circ}$, and toward the $\vec{k}_{i} \perp \vec{B}$ screen in a pyramid of half-angles $\sim 7.5^{\circ}$ and $12^{\circ}$ for the horizontal and vertical directions, respectively (see Fig. 1a). Upon turning the magnetic field ON the time-evolving patterns of the transmitted scattered light were recorded from the two screens using two synchronized digital cameras. Image analysis software was then used to convert the digital pictures into surface plots of the scattered light intensity. In a separate set of experiments, the screens were removed, and the time-dependent intensities of the light scattered in the forward direction for both $\vec{k}_{i} \perp \vec{B}$ and $\vec{k}_{i} \| \vec{B}$ were measured using two Newport 818-IS-1 integrating sphere detectors (entrance aperture of $\sim 1 \mathrm{~mm}$ ). The use of an integrating sphere detector minimizes any potential polarization dependence of the response of the Si detector itself. All measurements were conducted at ambient temperature $\left(\sim 22{ }^{\circ} \mathrm{C}\right)$, with no active temperature control.

\section{RESULTS AND DISCUSSION}

\subsection{Field OFF to Field ON transition}

A detailed analysis and discussion of the field-induced light-scattering patterns observed during the field $O F F$ to field $O N$ transition for the $\mathrm{Fe}_{3} \mathrm{O}_{4}$ ferrofluid can be found in Ref. [12]. For completeness, a brief summary of that discussion and results are included here. The field-induced light scattering patterns can be discussed within the framework and system of notations introduced by Kruse et al., as shown in Fig. 1.b. In this framework, the net result of the multiple scattering through the 10-mm long sample can be described in the 3D coordinate-space in terms of an effective scattering vector $\vec{h}$ or $\vec{h}^{\prime}$, respectively, where $\vec{h}=\left(h_{x}, h_{y}\right)=h(\cos \alpha, \sin \alpha)$ corresponds to the $\vec{k}_{i} \perp \vec{B}$ screen and $\vec{h}^{\prime}=\left(h_{x}^{\prime}, h_{y}^{\prime}\right)=h^{\prime}\left(\cos \alpha^{\prime}, \sin \alpha^{\prime}\right)$ to the $\vec{k}_{i} \| \vec{B}$ screen. In this representation, the scattered intensity $I(\vec{h})$ or $I\left(\vec{h}^{\prime}\right)$ is a function of the corresponding vector, $\vec{h}$ or $\vec{h}^{\prime}$.

Figures 2 and 3 show the scattered intensity patterns produced by two orthogonal $10 \mathrm{~mW}$, z-axis polarized red HeNe lasers on the $\vec{k}_{i} \perp \vec{B}$ screen (Fig. 2) and $\vec{k}_{i} \| \vec{B}$ screen (Fig. 3) for the field OFF to field ON transition in the two ferrofluid systems. With the ferrofluid samples having no prior history in a magnetic field, the field was turned $\mathrm{ON}$ at $\mathrm{t}=$ 0 , and the scattered intensities were recorded at regular time intervals for 60 minutes. Before applying the magnetic field (i.e. at $\mathrm{t}<0$, when $\mathrm{B}=0$ ), the intensity patterns for both ferrofluid systems in both $\vec{k}_{i} \perp \vec{B}$ and $\vec{k}_{i} \| \vec{B}$ directions are isotropic: at any give magnitude of the scattering vector h, they do not depend on the angle $\alpha$ or $\alpha^{\prime}$, respectively. This is a direct consequence of the random distribution of the particles in suspension in both ferrofluids in the absence of a magnetic field. However, upon turning the magnetic field $\mathrm{ON}$ at $\mathrm{t}=0$ ( 0 Oe to $400 \mathrm{Oe}$ transition, $<1 \mathrm{~s}$ ramp-up time), an anisotropy develops in the $\mathrm{Fe}_{3} \mathrm{O}_{4}$ ferrofluid in the $\vec{k}_{i} \perp \vec{B}$ direction (Fig. 2): at any given magnitude $h$ of the scattering vector, the intensity of light scattered perpendicular to the field, $I_{\perp}=I(h, \alpha= \pm \pi / 2)$ becomes larger than the intensity of light scattered parallel to the field, $I_{\|}=I(h, \alpha=0)=I(h, \alpha=\pi)$, i.e. $I_{\|}(h)<I_{\perp}(h)$. The pattern evolves monotonically in time toward a narrow, vertical line of light, thus presenting the general features of the anisotropic scattering pattern produced by a long thin cylinder or collection of parallel cylinders. ${ }^{11,30}$ This suggests the $\mathrm{Fe}_{3} \mathrm{O}_{4}$ nanoparticles are aggregating in chain-like structures aligned with the applied field. The intensity pattern observed along the $\vec{k}_{i} \perp \vec{B}$ direction in Fig. 2 for the $\mathrm{Fe}_{3} \mathrm{O}_{4}$ ferrofluid has a periodic angular dependence given by ${ }^{12}$

$$
I(h, \alpha)=I_{0}(h)\left\{1+A(h)\left(\sin ^{2 n} \alpha-0.5\right)\right\}
$$

where $I_{0}(h)$ denotes the radial mean value of the scattered intensity given by

$$
I_{0}(h)=\frac{I_{\perp}(h)+I_{\|}(h)}{2}
$$


$\mathrm{n}$ is a factor that depends on the magnitude of the scattering vector, $n=n(h)$, and

$$
A(h)=2 \frac{I_{\perp}(h)-I_{\|}(h)}{I_{\perp}(h)+I_{\|}(h)}
$$

$A(h)$ is a measure of the field-induced optical anisotropy which, in the steady state, depends on B.

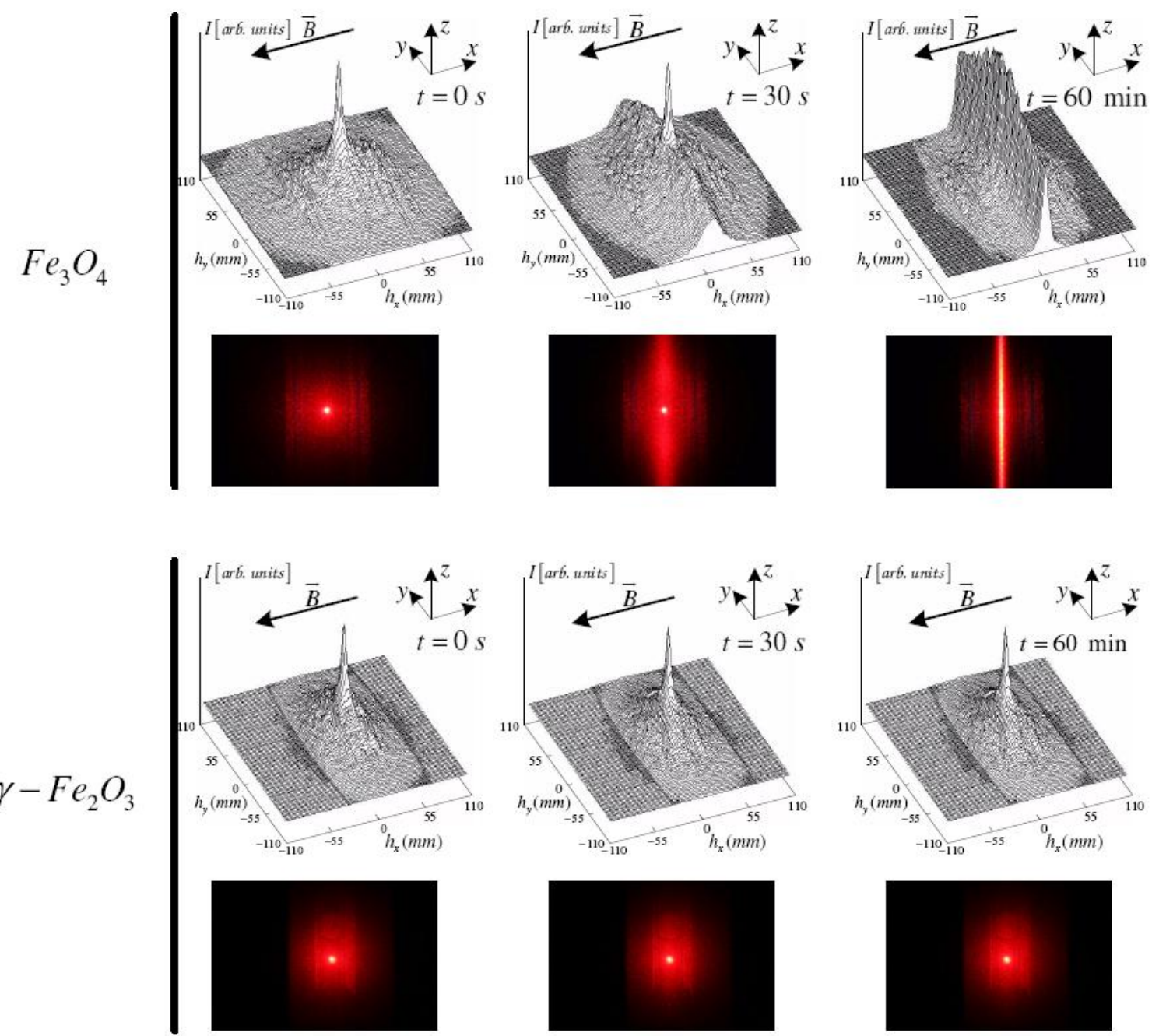

Fig. 2. Light scattering patterns and the corresponding surface plots for the scattered intensity on the $\vec{k}_{i} \perp \vec{B}$ screen from a $\mathrm{Fe}_{3} \mathrm{O}_{4}$ ferrofluid sample (top) and $\gamma-\mathrm{Fe}_{2} \mathrm{O}_{3}$ ferrofluid sample in alginate matrix (bottom) at representative instants of time. The samples had no prior history in the magnetic field. The magnetic field was turned $\mathrm{ON}$ at $\mathrm{t}=0$ with a rampup time of less than $1 \mathrm{~s}$, and kept at a steady dc value of 400 Oe throughout the entire experiment.

For a given strength of the steady dc magnetic field $\vec{B}, A(h)$ is a function of time, $A=A(h, t)=A_{h}(t)$. We found ${ }^{12}$ that the time-dependence of the optical anisotropy parameter $A_{h}(t)$, (see the inset in Fig. 4) extracted for the $\mathrm{Fe}_{3} \mathrm{O}_{4}$ ferrofluid from the time evolution of the anisotropic light scattering patterns in Fig. 2, generally resembled the field-induced optical transmittance results obtained by $\mathrm{Li}$ et al. ${ }^{9}$ and Martin et al. ${ }^{4}$ This time dependence of $A_{h}(t)$ showed a rapid change in the optical anisotropy at short times $(\mathrm{t}<30 \mathrm{~s})$, and a significantly slower change at longer times $(\mathrm{t}>5 \mathrm{~min})$, when $A(h, t)$ approaches a saturated value. We explain ${ }^{12}$ this time dependence for the $\mathrm{Fe}_{3} \mathrm{O}_{4}$ ferrofluid within the frame of a two-stage chain formation and coarsening process, as introduced by Halsey and Toor ${ }^{3}$ and expanded by Martin et al. ${ }^{4}$ 
and Furst and Gast. ${ }^{6}$, which predicts a faster process for chain formation at short times, and a slower process for chain coalescence into columns at longer times.

In contrast with the results for the $\mathrm{Fe}_{3} \mathrm{O}_{4}$ ferrofluid, it is apparent from Fig. 2 that no field-induced optical anisotropy can be detected for the $\gamma-\mathrm{Fe}_{2} \mathrm{O}_{3}$ ferrofluid sample in alginate matrix. Mathematically, this translates into the anisotropy parameter $A$ having an identical zero value at all scattering vectors and all times during the experiment. Physically, this suggests that no structural changes measurable by light scattering are being induced in this ferrofluid.
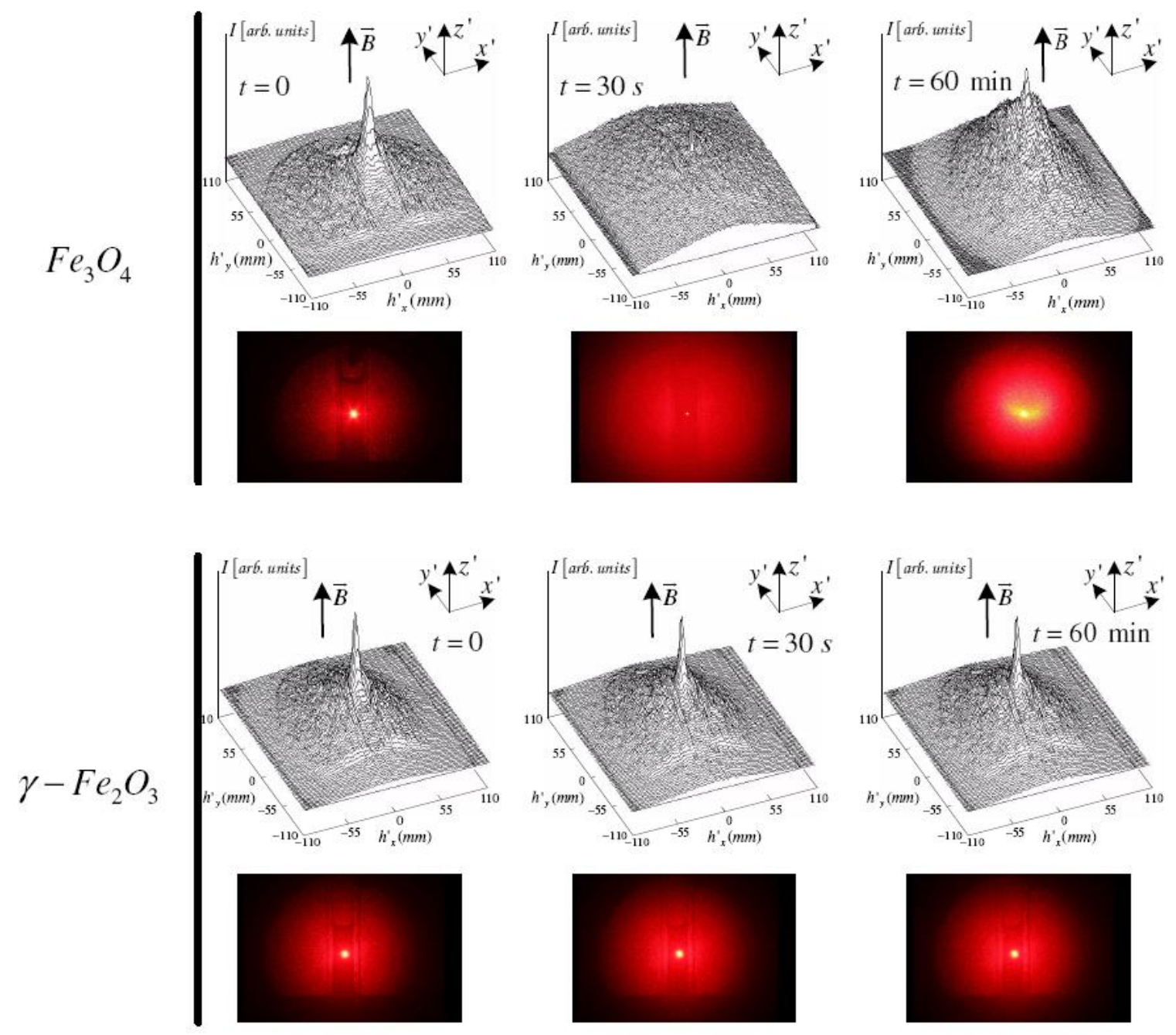

Fig. 3. Light scattering patterns and the corresponding surface plots for the scattered intensity on the $\vec{k}_{i} \| \vec{B}$ screen from a $\mathrm{Fe}_{3} \mathrm{O}_{4}$ ferrofluid sample (top) and $\gamma-\mathrm{Fe}_{2} \mathrm{O}_{3}$ ferrofluid sample (bottom) at representative instants of time. The samples had no prior history in the magnetic field. The magnetic field was turned $\mathrm{ON}$ at $\mathrm{t}=0$ with a ramp-up time of less than $1 \mathrm{~s}$, and kept at a steady dc value of 400 Oe throughout the entire experiment.

We now consider the scattering patterns on the $\vec{k}_{i} \| \vec{B}$ screen presented in Fig. 3, which were recorded, for each of the two ferrofluids, synchronously with the patterns on the $\vec{k}_{i} \perp \vec{B}$ screen. These measurements provide additional information for our investigation, beyond previous studies, ${ }^{4,} 7,9,24$ which confined their observations to steady-state and/or time dependent measurements for only one incident beam, propagating either parallel or perpendicular to the applied field. Similar to the observations on the $\vec{k}_{i} \perp \vec{B}$ screen, the patterns in Fig. 3 reveal a light-scattering response to the applied magnetic field only in the $\mathrm{Fe}_{3} \mathrm{O}_{4}$ ferrofluid. The $\mathrm{Fe}_{3} \mathrm{O}_{4}$ scattering patterns in Fig. 3, isotropic with respect to the direction of the scattering vector $\vec{h}^{\prime}$ and having circular symmetry about the applied magnetic field, reinforce the 
conclusion of chain formation and coarsening in the $\mathrm{Fe}_{3} \mathrm{O}_{4}$ ferrofluid suggested by the corresponding (synchronous) scattering patterns on the $\vec{k}_{i} \perp \vec{B}$ screen presented in Fig. 2 . The circular symmetry of the pattern on the $\vec{k}_{i} \| \vec{B}$ screen has been predicted ${ }^{11}$ and experimentally observed ${ }^{24}$ in other studies, and associated with the formation of chains along the field lines. The lack of such structures in the suspension of $\gamma-\mathrm{Fe}_{2} \mathrm{O}_{3}$ nanoparticles confined to an alginate matrix is reinforced by the identical lack of optical response to the applied field in both $\vec{k}_{i} \| \vec{B}$ and $\vec{k}_{i} \perp \vec{B}$ directions. The observation that diffusive chain formation seems to be suppressed in the $\gamma-\mathrm{Fe}_{2} \mathrm{O}_{3}$ ferrofluid is consistent with the expectation that the nanoparticle motion is limited by the alginate matrix in this system.

In order to include contribution to the scattering patterns from the scattering signal arising from both spherical and non-spherical nanoparticles partially aligning with the magnetic field, we use the analysis by Kruse et al. ${ }^{7}$ for a polydispersed system consisting of ellipsoidal particles with semi-axes lengths of $R_{i}, R_{i}$ and $k R_{i}\left(k_{i}>1\right)$. According to their analysis, the intensity scattered by a single ellipsoid depends on its extension in the direction of the scattering vector, and the $2 \mathrm{D}$ intensity pattern scattered by the ensemble of poly-dispersed ellipsoids is determined by the effective axis ratio ${ }^{7}$

$$
k_{\text {eff }}=\frac{E_{\|}}{E_{\perp}}
$$

Here, $E_{\|}$and $E_{\perp}$ are the effective semi-axes, and they represent ensemble averages of the particle extensions parallel and perpendicular to the applied magnetic field, respectively. The effective axis ratio $k_{\text {eff }}$ is a measure of both the particles orientation and asphericity. If the particles were completely oriented, $E_{\perp}=R_{\text {avg }}$ and $E_{\|}=k_{\text {avg }} R_{\text {avg }}$ would hold, where $R_{\text {avg }}$ and $k_{\text {avg }}$ are ensemble averages of $R_{i}$ and $k_{i}$; if the particles are randomly oriented, then $E_{\|}=E_{\perp}$. The effective axis ratio can be written in terms of the optical scattering anisotropy parameter $A$ as $^{7}$

$$
k_{\text {eff }}=\frac{E_{\|}}{E_{\perp}}=\left(\frac{I_{\perp}}{I_{\|}}\right)^{1 / 4}=\left(\frac{1+A / 2}{1-A / 2}\right)^{1 / 4}
$$

Equation (5) is valid at large scattering vectors, where the anisotropy parameter $A(h)$ no longer depends on the scatttering vector $h$, as discussed in Ref. [7]. From our experimental data for scattering in the $\vec{k}_{i} \perp \vec{B}$ direction by the TMAH-coated $\mathrm{Fe}_{3} \mathrm{O}_{4}$ ferrofluid presented in Fig. 2, we determine the corresponding time and scattering vector dependence of the anisotropy parameter $A$, and the corresponding time dependence of the effective axis ratio $k_{e} f$. Figure 4 shows the dependence of the anisotropy parameter $A$ on the magnitude of the scattering vector $h$ at $t=60$ min, when the ferrofluid has reached saturation. It can be observed form this $A$ vs. $h$ graph that the anisotropy parameter $A$ is indeed practically independent of $h$ for scattering vectors of magnitudes larger than approximately $80 \mathrm{~mm}$. This observation is also supported by the data in the inset to Fig 4, which shows the variation of $A$ with time. One can see in this inset how at large scattering vectors the saturation values of the anisotropy parameter $A$ converge toward a unique curve. This can also be correlated with the graphs in Fig. 5 which show the time dependence of the effective axis ratio, $k_{\text {eff }}$, for the $\mathrm{Fe}_{3} \mathrm{O}_{4}$ ferrofluid at several values of the scattering vector $h$. Once again, one can observe that for $h>80 \mathrm{~mm}$, the $k_{\text {eff }}$ vs. $t$ curves converge, indicative of the decreasing dependence of $A$, and thus of $k_{\text {eff }}$, on $h$. The $k_{\text {eff }}$ value for all values of the scattering vector $h$ change very rapidly within a few seconds of the application of the field, indicating a quick orientation of the non-spherical $\mathrm{Fe}_{3} \mathrm{O}_{4}$ particles with field lines, followed by their aggregation in oblong clusters. The $k_{\text {eff }}$ value becomes constant after this initial orientation showing the rapid re-orientation of the particles. This effect may be related to the anisotropy of self diffusion in ferrofluids which has been studied in relation to the oblate particle shape through dynamical mean field theory by Ilg et al. ${ }^{31,32}$. Ilg et al. found that for moderately interacting ferrofluids the diffusion in a direction perpendicular to the field, $D_{\perp}$, is greater than that parallel to the field lines, $D_{\Pi}$. The bottom horizontal line in Fig. 5, showing an identical value of $k_{\text {eff }}=1$ for all scattering vectors and at all times, corresponds to the $\gamma-\mathrm{Fe}_{2} \mathrm{O}_{3}$ ferrofluid, and is a reflection of the lack of field-induced anisotropy in this sample due to the random constrained distribution of the $\gamma-\mathrm{Fe}_{2} \mathrm{O}_{3}$ nanoparticles. This shows that although the magnetic moments of the $\gamma-\mathrm{Fe}_{2} \mathrm{O}_{3}$ nanoparticles 
confined to the alginate matrix may align with the magnetic field through Néel relaxation, they do not exhibit Brownian rotation.

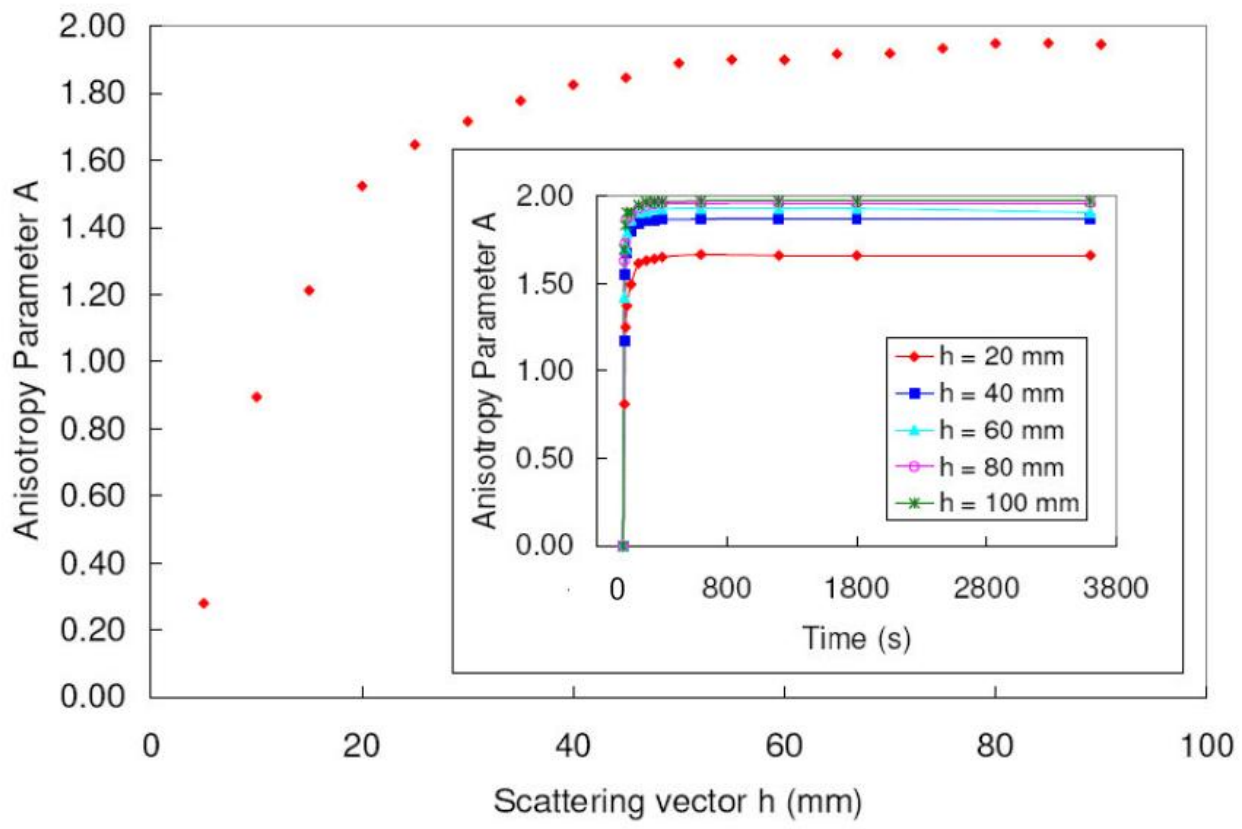

Fig. 4. The dependence of the anisotropy parameter $A$ for the $\mathrm{Fe}_{3} \mathrm{O}_{4}$ ferrofluid on the magnitude of the scattering vector $h$ at saturation $(t=60 \mathrm{~min})$. The inset presents the time dependence of the anisotropy parameter A for several values of the scattering vector $h$. A convergence of the $A$ vs. $t$ curves at large scattering vectors h can be observed.

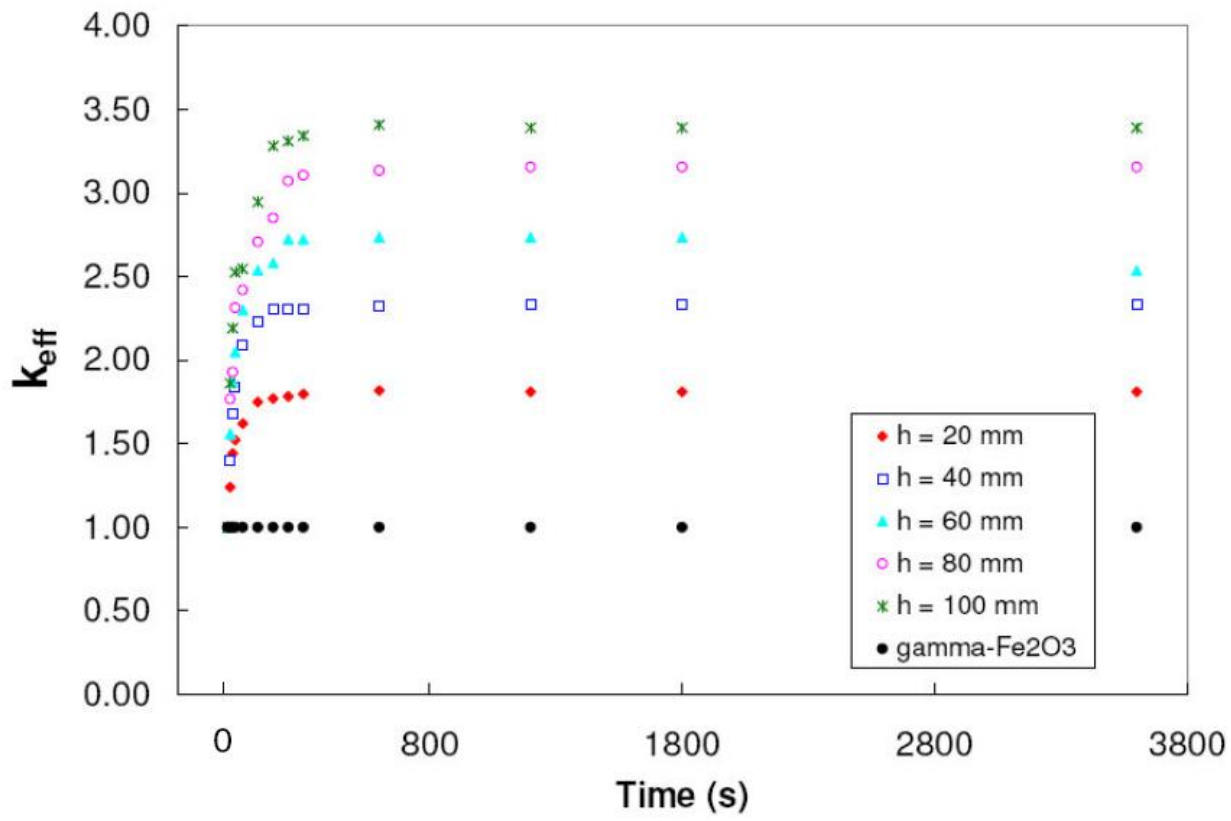

Fig. 5. The dependence of the effective axis ratio, $k_{\text {eff }}$, for the $\mathrm{Fe}_{3} \mathrm{O}_{4}$ ferrofluid, at several values of the scattering vector $\mathrm{h}$. The bottom horizontal line at $k_{\text {eff }}=1$ corresponds to the $\gamma-\mathrm{Fe}_{2} \mathrm{O}_{3}$ ferrofluid and results from the lack of field-induced optical anisotropy. 


\subsection{Field ON to Field OFF transition}

Rather different optical anisotropy patterns are exhibited by the $\mathrm{Fe}_{3} \mathrm{O}_{4}$ and $\gamma-\mathrm{Fe}_{2} \mathrm{O}_{3}$ ferrofluid samples when the field is switched OFF. Figures $6 \mathrm{a}$ and $6 \mathrm{~b}$ show the light scattering patterns and the corresponding surface plots for the scattered intensity on the $\vec{k}_{i} \perp \vec{B}$ screen (6a) and $\vec{k}_{i} \| \vec{B}$ screen (6b), at representative instants of time, for the $\mathrm{Fe}_{3} \mathrm{O}_{4}$ sample. Prior to turning OFF the magnetic field at $\mathrm{t}=0$, the sample had been held in a steady 400 Oe DC field for 60 minutes. We find that the system does not become isotropic almost instantaneously, as observed by Martin et al. ${ }^{4}$ Although the dissociation and segregation of the chains appears to occur much more rapidly than the aggregation process, particularly at short times $(\mathrm{t}<30 \mathrm{~s})$, it takes about five minutes for the ferrofluid to completely recover the isotropic state, during which time the $\mathrm{Fe}_{3} \mathrm{O}_{4}$ ferrofluid evolves through states producing different but anisotropic light scattering patterns in both $\vec{k}_{i} \perp \vec{B}$ and $\vec{k}_{i} \| \vec{B}$ directions. In addition, one can observe interesting angular fluctuations in the pattern, which are significantly stronger in the $\vec{k}_{i} \perp \vec{B}$ direction.

a)
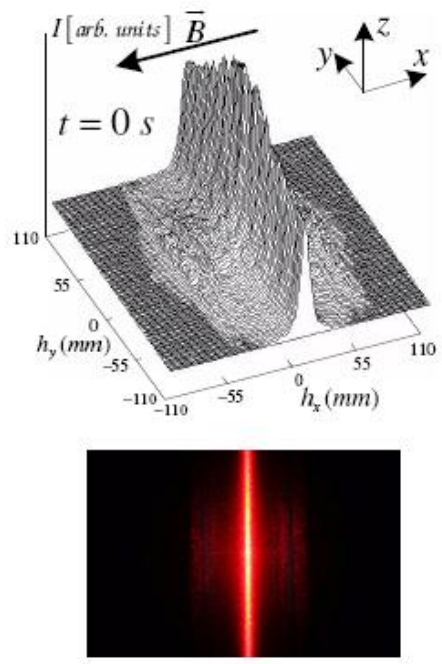

b)
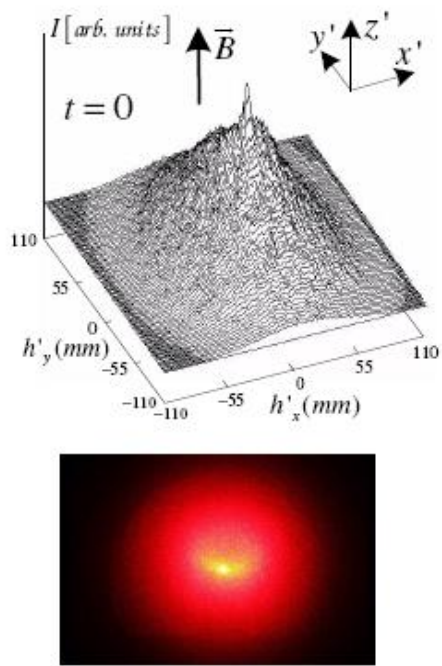
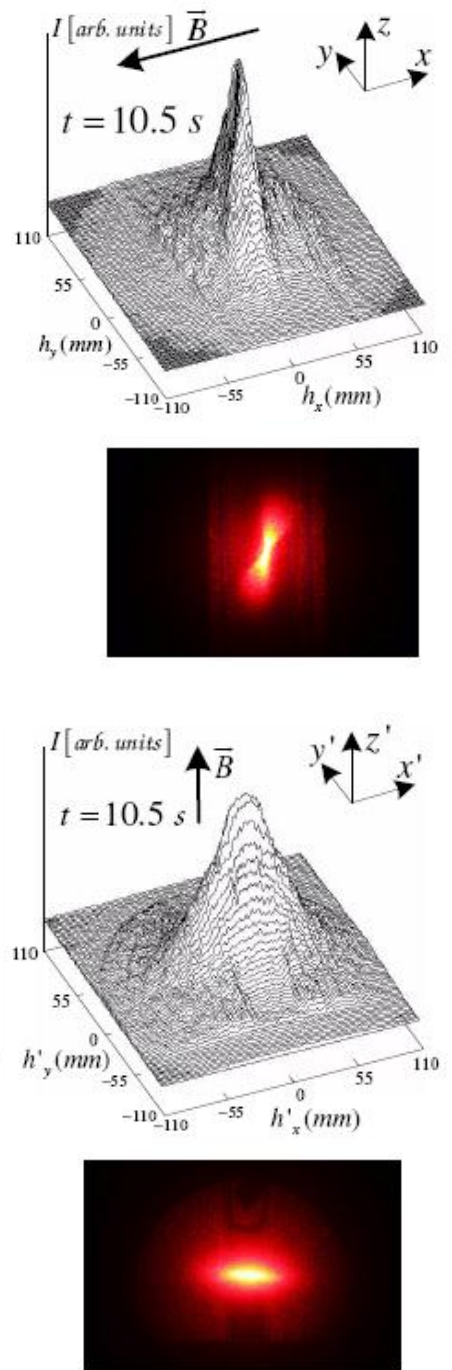
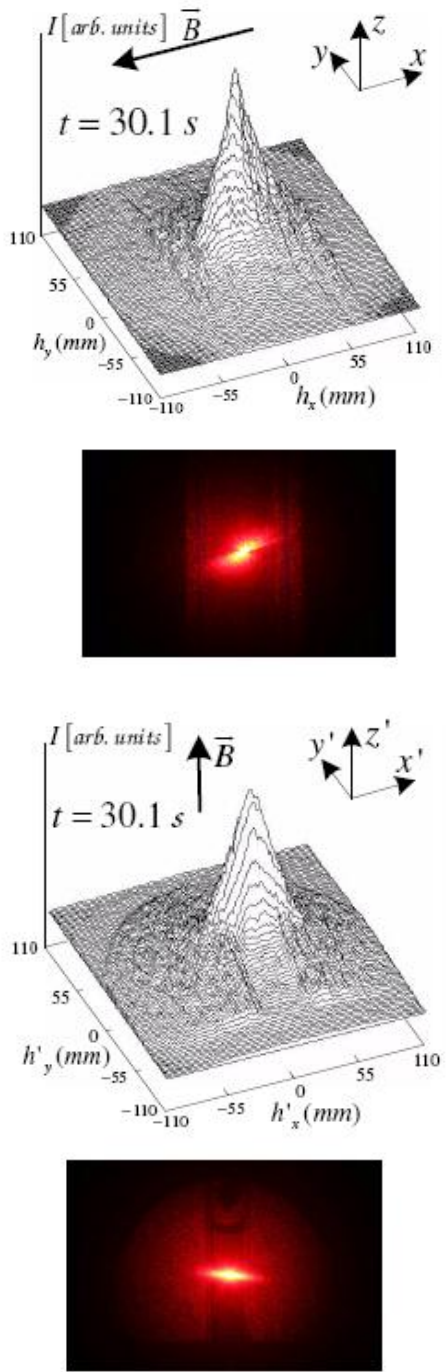

Fig. 6. Light scattering patterns from a $\mathrm{Fe}_{3} \mathrm{O}_{4}$ ferrofluid sample and the corresponding surface plots for the scattered intensity on the $\vec{k}_{i} \perp \vec{B}$ screen (6a) and $\vec{k}_{i} \| \vec{B}$ screen (6b), at representative instants of time after the field ON to field OFF transient of dc magnetic field. Prior to turning OFF the magnetic field at $\mathrm{t}=0$, the sample has been held in a steady 400 Oe de field for 60 minutes. The field ramps down from field ON to field OFF in less than one second. 
This rotation of the patterns suggests a rotational nature of the diffusion during the segregation and dissociation of the columns and chains into smaller structures. Rotational diffusion has indeed been observed in ferrofluids, but only when exposed to ac magnetic fields. ${ }^{33} \mathrm{We}$ find this result rather remarkable that such angular intensity fluctuations can be produced by a rapidly decaying dc magnetic field applied to a structurally ordered ferrofluid. In contrast, but similar to the field OFF to field $O N$ transition, no measurable change in the optical scattering patterns could be observed for the $\gamma$ $\mathrm{Fe}_{2} \mathrm{O}_{3}$ suspension when the field was switched OFF after having held the sample in a steady dc fields for times as long as two hours. Although both $\mathrm{Fe}_{3} \mathrm{O}_{4}$ and $\mathrm{g}-\mathrm{Fe}_{2} \mathrm{O}_{3}$ have similar inverse spinel structure, they exhibit different physical behavior under the influence of an applied dc magnetic field. The knowledge of their behavior is therefore crucial in biomedical applications such as targeted drug delivery, MRI and cell separation, where the ferrofluids are subjected to external dc magnetic fields.

The results of this light scattering investigation are consistent with our previous measurements of ac magnetic susceptibility, magnetic relaxation and dissipative heating in ferrofluids ${ }^{27}$ performed on the same two ferrofluid systems as the ones included in this work. The results from the present investigation, in particular the observation of angular diffusion during the field $O N$ to field OFF transition, when compared with our previous study, seem to suggest that chain formation and dissociation in a ferrofluid in dc magnetic field and the magnitude of the hyperthermia effect in the same ferrofluid in an ac magnetic field could be correlated. However, further study is needed to better understand the kinematics of the de-coarsening of the thick columns of chains, segregation of longer chains into the smaller segments, and finally into individual nanoparticles, as well as the correlation of this kinematics with the ac Brownian relaxation in the ferrofluid.

\section{CONCLUSIONS}

We have presented the results of a comparative time-dependent light scattering investigation for two ferrofluid systems: an aqueous suspension of 6-nm-size TMAH-surfacted $\mathrm{Fe}_{3} \mathrm{O}_{4}$ nanoparticles and an aqueous suspension of 6-nmsize $\gamma-\mathrm{Fe}_{2} \mathrm{O}_{3}$ nanoparticles precipitated in alginate hydrogel matrix. The dc magnetic-field-induced light-scattering patterns produced in transmission by two orthogonal light beams propagating through the ferrofluid sample reveal a strong field-induced anisotropy in the $\mathrm{Fe}_{3} \mathrm{O}_{4}$ ferrofluid, but no significant change in the $\gamma-\mathrm{Fe}_{2} \mathrm{O}_{3}$ ferrofluid. We describe the induced optical anisotropy in terms of a time-dependent optical anisotropy parameter $A$ which, for the $\mathrm{Fe}_{3} \mathrm{O}_{4}$ ferrofluid, increases rapidly at short times $(\mathrm{t}<30 \mathrm{~s})$ and much more slowly at longer times $(\mathrm{t}>5$ min), toward a saturated value. We attribute the observed optical anisotropy in the $\mathrm{Fe}_{3} \mathrm{O}_{4}$ ferrofluid to a field-induced structural anisotropy and we explain it within the framework of the existing models related to chain formation and coarsening in ferrofluids. No induced anisotropy was observed for the aqueous suspension of $\gamma-\mathrm{Fe}_{2} \mathrm{O}_{3}$ nanoparticles precipitated in alginate hydrogel matrix. We attribute this lack of induced anisotropy to the static random distribution of $\gamma$ - $\mathrm{Fe}_{2} \mathrm{O}_{3}$ nanoparticles in the alginate matrix. The field $O N$ to field OFF transition reveals that the segregation and dissociation of the chain-like structures in the $\mathrm{Fe}_{3} \mathrm{O}_{4}$ ferrofluid occurs approximately ten times faster than the aggregation process. The interesting feature of angular fluctuations of the anisotropic scattering patterns after switching OFF the field suggests the presence of an angular field and/or rotational diffusion during the segregation of the chains.

\section{ACKNOWLEDGMENT}

The authors at Wayne State University acknowledge the support of their work by the donors of the American Chemical Society (PRF\# 46160-G10), Richard J. Barber Funds for Interdisciplinary Research and the Institute for Manufacturing Research at Wayne State University.

\section{REFERENCES}

[1] Alexiou, C, .Schmid, R. J., Jurgons, R., Kremer, M., Wanner, G., Bergemann, C., Huenges, E., Nawroth, T., Arnold, W., Parak, F. G., "Targeting cancer cells: magnetic nanoparticles as drug carriers," Eur. Biophys. J. 35, 446-450 (2006).

[2] Fortin, J.-P., Gazeau, F., Wilhelm, C., "Intracelular heating of living cells through Néel relaxation of magnetic nanoparticles," Eur. Biophys. J. 37, 223-228 (2008). 
[3] Halsey, T.C., and Toor, W., "Structure of Electrorheological Fluids," Phys. Rev. Lett. 65, 2820-2823 (1990).

[4] Martin, J. E., Hill, K M., and Tigges, C. P., "Magnetic-field-induced optical transmittance in colloidal suspensions," Phys. Rev. E 59, 5676-5692 (1999).

[5] Martin, J. E., Odinek,, J., Halsey, T. C., and Kamien, R., "Structure and dynamics of electrorheological fluids," Phys. Rev. E 57, 756 (1998).

[6] Furst, E. M., and Gast, A. P., "Dynamics and lateral interactions of dipolar chains,” Phys. Rev. E 62, 6916-6925 (2000).

[7] Kruse, T., Krauthauser, H. G., Spanoudaki, A., and Pelster, R., "Agglomeration and chain formation in ferrofluids: Two-dimensional x-ray scattering," Phys. Rev B 67, 094206 (2003).

[8] Fraden, S., Hurd, A. J., and Meyer, R. B., "Electric-Field-Induced Association of Colloidal Particles,” Phys. Rev. Lett. 63, 2373-2376 (1989).

[9] Li, J., Liu, X. D., Lin, Y.Q., Huang, Bai, Y. L., "Relaxation behavior measuring of transmitted light through ferrofluids film," Appl. Phys. B 82, 81-84 (2006).

[10] Melle, S., Calderon, O. G., Rubio, M. A., and Fuller, G. G., "Microstructure evolution in magnetorheological suspensions governed by Mason number," Phys. Rev. E 68, 041503 (2003).

[11] S. Melle, Ph.D. Dissertation and references therein, Spanish National Open University (UNED), (2002).

[12] Rablau, C., Vaishnava, P.P., Sudakar, C., Tackett, R., Lawes, G., and Naik, R., "Magnetic-field-induced optical anisotropy in ferrofluids: A novel time-dependent light scattering investigation," submitted to Phys. Rev E (2008).

[13] Fannin, P. C., and Charles, S. W., "The study of a ferrofluid exhibiting both Brownian and Néel relaxation," J. Phys. D: Appl. Phys. 22, 187-191 (1989).

[14] Smith, D.A., and Stokes, K. L., "Discrete dipole approximation for magneto-optical scattering calculations," Optics Express 14, 5746-5754 (2006).

[15] Huang, J. P., Wang, Z. W., and Holm, C., "Computer simulations of the structure of colloidal ferrofluids," Phys. Rev. E 71, 061203 (2005).

[16] Ivanov, A. O., Wang, Z., and Holm, C., "Applying the chain formation model to magnetic properties of aggregated ferrofluids," Phys. Rev. E 69, 031206 (2004).

[17] Mendelev, V. S., and Ivanov, A. O., "Ferrofluid aggregation in chains under the influence of a magnetic field," Phys. Rev. E 70, 051502 (2004).

[18] Kruse, T., Spanoudaki, A. and Pelster, R., "Monte Carlo simulations of polydisperse ferrofluids: Cluster and fielddependent microstructure," Phys. Rev B 68, 054208 (2003).

[19] Miguel, M.C. and Pastor-Satorras R., "Kinetic growth of field-oriented chains in dipolar colloidal solutions," Phys. Rev. E 59, 826-834 (1999).

[20] Heinrich, D., Goni, A. R., and Thomsen, C., "Dynamics of magnetic-field-induced clustering in ionic ferrofluids from Raman scattering," J. Chem. Phys. 126, 124701 (2007).

[21] Morais, P. C., Skeff Neto, K., Bakuzis, A. F., Da Silva, M. F., and Buske, N. "Birefringence of magnetite-based magnetic fluids: The effect of the surface coating layer", IEEE Trans. Magn. 38, 3228-3230 (2002).

[22] Donatini, F., Jamon, D., Monin, J., and Neveu, S., "Experimental investigation of longitudinal magneto-optic effects in four ferrite ferrofluids in visible-near infrared spectrum," IEEE Trans. Magn. 35, 4311-4317 (1999).

[23] Payet, B., Donatini, F., and Noyel, G., "Longitudinal magneto-optical study of Brown relaxation in ferrofluids: dynamic and transient methods. Application,” J. Magn. Magn. Mater. 201, 207-210 (1999).

[24] Hwang, Y. H., and Wu, X.-I., "Quasi-two-dimensional domain structures of magnetic particles in a static field," Phys. Rev. E 49, 3102-3108 (1994).

[25] Da Silva, M. F., and Figueiredo Neto, A. M., "Optical- and x-ray-scattering studies of ionic ferrofluids of $\mathrm{MnFe}_{2} \mathrm{O}_{4}$, $\gamma-\mathrm{Fe}_{2} \mathrm{O}_{3}$ and $\mathrm{CoFe}_{2} \mathrm{O}_{4}$," Phys. Rev. E 48, 4483-4491 (1993).

[26] Ginder, J. M., "Diffuse optical probes of particle motion and structure formation in an electrorheological fluid," Phys. Rev. E 47, 3418-3429 (1993).

[27] Vaishnava, P.P., Tackett, R., Dixit, A., Sudakar, C., Naik, R., and Lawes, G., "Magnetic relaxation and dissipative heating in ferrofluids," J. Appl Phys. 102, 063914 (2007).

[28] E. Kroll, F.M. Winnik and R.F. Ziolo, "In situ preparation of nanocrystalline $\gamma-\mathrm{Fe}_{2} \mathrm{O}_{3}$ in iron (II) cross-linked alginate gels," Chem. Mater. 8, 1594-1596 (1996).

[29] Wenger, L.E., Tsoi, G.M., Vaishnava, P.P., Senaratne, U., Buc, E.C., Naik, R., and Naik, V.M., "Magnetic properties of $\gamma-\mathrm{Fe}_{2} \mathrm{O}_{3}$ nanoparticles precipitated in alginate hydrogels" IEEE Trans. Magn., 2008 (accepted for publication)

[30] van de Hulst, H.C. [Light Scattering by Small Particles], Dover Publications, New York, (1981) 
[31] Ilg, P., “Anisotropic diffusion in nematic liquid crystals and in ferrofluids,” Phys. Rev E 71, 051407 (2005).

[32] Ilg, P., "Anisotropic self-diffusion in ferrofluids studied via Brownian dynamics simulations," Phys. Rev E 72, 031504 (2005).

[33] Erne, B., Butter, K., Kuiper, B. W. M., and Vroege, G. J., "Rotational diffusion in iron ferrofluids,” Langmuir, 19, 8218 (2003). 